\title{
INTERNATIONAL UNION OF STUDENTS (IUS) UNION INTERNATIONALE DES ETUDIANT(E)S (UIE)
}

P.O. Box 58

17 th November Street

11001 Prague 01 (Czechoslovakia)

Tel: (422) 2312812

Fax: (422) 2316100

Telex: 122812 iusc

Cables: unistud prague

Secretary-General: Giorgos Michaelides

An independent international non-governmental organization registered in the Czech and Slovak Federal Republic. The Constitution of the IUS was amended at an Extraordinary Congress, held in Prague on April 7-10 1991. IUS groups together the national unions of students from over 110 countries. It strives for education, academic freedom, student rights, for the role of students in social affairs (peace, environment, solutions to global problems, etc.); and organizes seminars and meetings. Its specialized structures include: International Student Research Centre (ISRC); International Student Travel Bureau (ISTB); International Student Scheme(ISES); International StudentCentre for Cultural Activities (ISCCA); International Physical Education and Sport Centre (ISPESC).

Principal publications: World Student News (monthly); Democratization of Education (DE); Student Life; IUS Newsletter (in english, french and spanish).

L'Union internationale des étudiants (UIE) est une organisation internationale non-gouvernementale enregistrée en République tchèque et slovaque fédérative. $L a$ constitution de l'UIE a été modifiée lors du congrès extraordinaire qui s' est tenu du 7 au 10 avril 1991 à Prague. L'Union regroupe les unions nationales d'étudiants de plus de 110 pays. L'UIE défend l'éducation, la liberté académique, les droits de l'étudiant, la place que doivent tenir les étudiants dans les affaires sociales (la paix, l' environnement, la solution à des problèmes d'ordre général, etc.). Elle organise des séminaires et des réunions. Ses organes spécialisés comprennent les organisations internationales suivantes: Centre Etudiant International de Recherche (CEIR); "International Student Travel Bureau" (ISTB); "International Student Exchange Scheme" (ISES); "International Student Centre for Cultural Activities" (ISCCA); "International Student Physical Education and Sports Centre" (ISPESC).

Publications principales: "World Student News" (mensuel); "Democratization of Education (DE)"; "Student Life"; "IUS Newsletter" (les publications sont en anglais, français et espagnol). 